CREAT. MATH. INFORM.

Volume 29 (2020), No. 2,

Pages $243-250$

\title{
Weighted composition operators from Bloch-type into Bers-type spaces
}

\author{
HAMID VAEZI and MOHAMAD NAGHLISAR
}

ABSTRACT. In this paper we consider the weighted composition operator $u C_{\varphi}$ from Bloch-type space $B^{\alpha}$ into Bers-type space $H_{\beta}^{\infty}$, in three cases, $\alpha>1, \alpha=1$ and $\alpha<1$. We give the necessary and sufficient conditions for boundedness and compactness of the above operator.

\section{INTRODUCTION}

Let $\mathbb{D}$ be the open unit disc in the complex plane $\mathbb{C}$ and $H(\mathbb{D})$ the space of analytic functions on $\mathbb{D}$. An analytic function $f$ on $\mathbb{D}$ is said to belong to the Bloch-type space $B^{\alpha}(0<\alpha<\infty)$, if

$$
\|f\|_{\alpha}=\sup _{z \in \mathbb{D}}\left(1-|z|^{2}\right)^{\alpha}\left|f^{\prime}(z)\right|<\infty .
$$

The expression $\|\cdot\|_{\alpha}$ defines a seminorm, while the natural norm is given by $\|f\|=$ $|f(0)|+\|f\|_{\alpha}$. This norm makes Bloch-type space $B^{\alpha}$ into a Banach space.

Let $u$ be an analytic function on $\mathbb{D}$ and $\varphi$ a nonconstant analytic self-map of $\mathbb{D}$. We define a linear operator $u C_{\varphi}$ on $H(\mathbb{D})$ by

$$
u C_{\varphi} f=u(f \circ \varphi) .
$$

This operator is called weighted composition operator. The operator $u C_{\varphi}$ can be regarded as a generalization of a multiplication operator and a composition operator. In case $u \equiv 1$, $u C_{\varphi}$ reduses to the composition operator $C_{\varphi}$ and when $\varphi(z)=z, u C_{\varphi}$ will be the multiplication operator $M_{u}$. For general back ground on composition operators, we refer [2, 9] and references therein.

Boundedness and compactness of composition operator on the Bers-type space were described by He Weixiang and Jiang Lijian in [12]. Zengjuan Lou in [6] characterized the boundedness and compactness of the composition operators between Bloch-type spaces. Several characterizations for the boundedness and compactness of the weighted composition operators from Bloch-type spaces to nth weighted-type spaces, also, some estimates for their essential norms are given by Li, Abbasi and Vaezi in [5]. Vaezi and Houdfar in [11], characterized the boundedness and compactness of composition and weighted composition operators from Bloch-type to Besov-type spaces.

The weighted composition operators acting on various spaces of analytic functions has been studied by many authors. For example, $u C_{\varphi}$ was studied by Ohno, Stroethoff and Zhao in [7], where the boundedness and compactness of $u C_{\varphi}$ between Bloch-type spaces are investigated. Collona and Li in [1] characterized the bounded and the compact weighted composition operators from the Besove space into Bloch space and Kumar in [4] characterized the boundedness and compactness of $u C_{\varphi}$ between Drichlet-type spaces. In

Received: 12.08.2019. In revised form: 20.02.2020. Accepted: 01.03.2020

2010 Mathematics Subject Classification. 47B33, 30H30, 30H99.

Key words and phrases. Weighted composition operator, Bloch-type space, Bers-type space, Boundedness, compactness.

Corresponding author: Hamid Vaezi; hvaezi@tabriz.ac.ir 
[8], adjoints of rationally induced weighted composition operators on the Hardy, Bergman and Dirichlet spaces was studied by Salaryan and Vaezi. Boundedness and compactness of this operator on weak vector-valued Bergman spaces and Hardy spaces are investigated by Hassanlou, Vaezi and Wang in [3]. In this paper, we study the operator $u C_{\varphi}$ from the Bloch-type space into the Bers-type space. We characterize boundedness and compactness of $u C_{\varphi}: B^{\alpha} \rightarrow H_{\beta}^{\infty}$ in three case, for $\alpha>1$ in section 2 , for $\alpha=1$ in section 3 and for $0<\alpha<1$ in section 4 . We need the following lemma (see [10]).

Lemma 1.1. Let $f \in B^{\alpha}, 0<\alpha<\infty$. Then

where $C$ is a constant.

$$
|f(z)| \leq C \begin{cases}\|f\|_{\alpha} & \alpha \in(0,1), \\ \|f\|_{\alpha} \operatorname{Ln} \frac{e}{1-|z|^{2}} & \alpha=1, \\ \|f\|_{\alpha} \frac{1}{\left(1-|z|^{2}\right)^{\alpha-1}} & \alpha>1 .\end{cases}
$$

Throughout this paper, constants are denoted by $C$, they are positive and may differ from one occurrence to the other.

2. BOUNDEDNESS AND COMPACTNESS OF $u C_{\varphi}: B^{\alpha} \rightarrow H_{\beta}^{\infty}$ FOR $\alpha>1$

In this section, we characterize the boundedness and compactness of $u C_{\varphi}: B^{\alpha} \rightarrow H_{\beta}^{\infty}$, when $\alpha>1$.

Theorem 2.1. Let $u$ be an analytic function on $\mathbb{D}, \varphi$ an analytic self-map of $\mathbb{D}, \alpha$ and $\beta$ positive real numbers and $\alpha>1$. Then $u C_{\varphi}: B^{\alpha} \rightarrow H_{\beta}^{\infty}$ is bounded if and only if

$$
\sup _{z \in \mathbb{D}} \frac{|u(z)|\left(1-|z|^{2}\right)^{\beta}}{\left(1-|\varphi(z)|^{2}\right)^{\alpha-1}}<\infty .
$$

Proof. First we obtain sufficiency. For a function $f \in B^{\alpha}$, we have

$$
\begin{aligned}
\sup _{z \in \mathbb{D}}\left(1-|z|^{2}\right)^{\beta}\left|u C_{\varphi} f(z)\right| & =\sup _{z \in \mathbb{D}}|u(z)|\left(1-|z|^{2}\right)^{\beta}|f(\varphi(z))| \\
& \leq \sup _{z \in \mathbb{D}}|u(z)|\left(1-|z|^{2}\right)^{\beta} \frac{C|| f \|_{\alpha}}{\left(1-|\varphi(z)|^{2}\right)^{\alpha-1}} \\
& =C \sup _{z \in \mathbb{D}} \frac{|u(z)|\left(1-|z|^{2}\right)^{\beta}}{\left(1-|\varphi(z)|^{2}\right)^{\alpha-1}}\|f\|_{\alpha} \\
& =C\|f\|_{\alpha} .
\end{aligned}
$$

In the above inequality we use the Lemma 1.1 for $\alpha>1$. Thus $u C_{\varphi}$ maps $B^{\alpha}$ boundedly into $H_{\beta}^{\infty}$.

Now, suppose that $u C_{\varphi}: B^{\alpha} \rightarrow H_{\beta}^{\infty}$ is bounded. For fixed $z_{0} \in \mathbb{D}$, consider the function $f_{0}$ defined by

$$
f_{0}(z)=\frac{1}{\left(1-z \overline{\varphi\left(z_{0}\right)}\right)^{\alpha-1}},
$$

for $z \in \mathbb{D}$.

It is easy to check that $f_{0} \in B^{\alpha}$ and then

$$
\begin{aligned}
|u(z)|\left(1-|z|^{2}\right)^{\beta} \frac{1}{\left|1-\varphi(z) \overline{\varphi\left(z_{0}\right)}\right|^{\alpha-1}} & =|u(z)|\left(1-|z|^{2}\right)^{\beta}\left|f_{0}(\varphi(z))\right| \\
& =\left(1-|z|^{2}\right)^{\beta}\left|u C_{\varphi} f_{0}(z)\right| \\
& \leq\left\|u C_{\varphi} f_{0}\right\|_{\beta} \leq C|| f_{0} \|_{\alpha}<\infty
\end{aligned}
$$


So for $z_{0} \in \mathbb{D}$,

$$
\frac{\left|u\left(z_{0}\right)\right|\left(1-\left|z_{0}\right|^{2}\right)^{\beta}}{\left(1-\left|\varphi\left(z_{0}\right)\right|^{2}\right)^{\alpha-1}}<\infty .
$$

Since $z_{0}$ is arbitrary, hence

$$
\sup _{z \in \mathbb{D}} \frac{|u(z)|\left(1-|z|^{2}\right)^{\beta}}{\left(1-|\varphi(z)|^{2}\right)^{\alpha-1}}<\infty .
$$

Theorem 2.2. Let $u$ be an analytic function on $\mathbb{D}, \varphi$ an analytic self-map of $\mathbb{D}, \alpha$ and $\beta$ positive real numbers and $\alpha>1$. Then $u C_{\varphi}: B^{\alpha} \rightarrow H_{\beta}^{\infty}$ is compact if and only if $u \in H_{\beta}^{\infty}$ and

$$
\lim _{|\varphi(z)| \rightarrow 1^{-}} \frac{|u(z)|\left(1-|z|^{2}\right)^{\beta}}{\left(1-|\varphi(z)|^{2}\right)^{\alpha-1}}=0 .
$$

Proof. Suppose that

$$
\lim _{|\varphi(z)| \rightarrow 1} \frac{|u(z)|\left(1-|z|^{2}\right)^{\beta}}{\left(1-|\varphi(z)|^{2}\right)^{\alpha-1}}=0 .
$$

By the assumption, for every $\varepsilon>0$, There exist a $\delta \in(0,1)$, such that

$$
\frac{|u(z)|\left(1-|z|^{2}\right)^{\beta}}{\left(1-|\varphi(z)|^{2}\right)^{\alpha-1}}<\varepsilon,
$$

whenever $\delta<|\varphi(z)|<1$. To prove the compactness of $u C_{\varphi}$, assume that $\left(f_{k}\right)_{k \in \mathbb{N}}$ is a bounded sequence in $B^{\alpha}$ such that $\left\|f_{k}\right\|_{\alpha} \leq 1$ and converges to zero uniformly on compact subsets of $\mathbb{D}$. We show that $\left\|u C_{\varphi} f_{k}\right\|_{\beta} \rightarrow 0$.

if $|\varphi(z)|>\delta$, then by (2.3),

$$
\begin{aligned}
\left\|u C_{\varphi} f_{k}\right\|_{\beta} & =\sup _{z \in \mathbb{D}}\left(1-|z|^{2}\right)^{\beta}\left|u C_{\varphi} f_{k}(z)\right| \\
& =\sup _{z \in \mathbb{D}} \frac{|u(z)|\left(1-|z|^{2}\right)^{\beta}}{\left(1-|\varphi(z)|^{2}\right)^{\alpha-1}} \mid f_{k} \|_{\alpha} \\
& <\varepsilon\left\|f_{k}\right\|_{\alpha} \leq \varepsilon .
\end{aligned}
$$

Now consider $|\varphi(z)| \leq \delta$. We have

$$
\left\|u C_{\varphi} f_{k}\right\|_{\beta}=\sup _{z \in \mathbb{D}}|u(z)|\left(1-|z|^{2}\right)^{\beta}\left|f_{k}(\varphi(z))\right| .
$$

Since $u \in H_{\beta}^{\infty}$, so $\left\|u C_{\varphi} f_{k}\right\|_{\beta} \rightarrow 0$.

Conversely, note that $u=u C_{\varphi} 1 \in H_{\beta}^{\infty}$. Now we are going to prove that (2.2) is also necessary condition for compactness of $u C_{\varphi}$. Suppose that $\left(z_{k}\right)_{k \in \mathbb{N}}$ is a sequence in $\mathbb{D}$ such that $\left|\varphi\left(z_{k}\right)\right| \rightarrow 1$ as $k \rightarrow \infty$.

Consider the functions $f_{k}$ defined by

$$
f_{k}(z)=\frac{1-\left|\varphi\left(z_{k}\right)\right|^{2}}{\left(1-z \overline{\varphi\left(z_{k}\right)}\right)^{\alpha}} \quad \text { for } \quad z \in \mathbb{D} .
$$


Clearly $f_{k} \rightarrow 0$ uniformly on compact subsets of $\mathbb{D}$, and

$$
\begin{aligned}
\left|f_{k}^{\prime}(z)\right| & =\frac{\alpha\left(1-\left|\varphi\left(z_{k}\right)\right|^{2}\right)\left|\overline{\varphi\left(z_{k}\right)}\right|}{\left|1-z \overline{\varphi\left(z_{k}\right)}\right|^{\alpha+1}} \\
& \leq \frac{\alpha\left(1-\left|\varphi\left(z_{k}\right)\right|^{2}\right)}{\left(1-|z|\left|\varphi\left(z_{k}\right)\right|\right)^{\alpha+1}} \\
& =\frac{\alpha\left(1-\left|\varphi\left(z_{k}\right)\right|^{2}\right)}{\left(1-|z|\left|\varphi\left(z_{k}\right)\right|\right)^{\alpha}\left(1-|z|\left|\varphi\left(z_{k}\right)\right|\right)} \\
& \leq \frac{\alpha\left(1+\left|\varphi\left(z_{k}\right)\right|\right)}{(1-|z|)^{\alpha}} \\
& \leq \frac{2 \alpha}{(1-|z|)^{\alpha}}<\infty .
\end{aligned}
$$

So,

$$
\begin{aligned}
\left\|f_{k}\right\|_{\alpha} & =\sup _{z \in \mathbb{D}}\left(1-|z|^{2}\right)^{\alpha}\left|f_{k}^{\prime}(z)\right| \\
& \leq \sup _{z \in \mathbb{D}} \frac{2 \alpha\left(1-|z|^{2}\right)^{\alpha}}{(1-|z|)^{\alpha}} \\
& \leq 2 \alpha \cdot 2^{\alpha} \\
& =\alpha 2^{\alpha+1}<\infty .
\end{aligned}
$$

Hence, $\left(\left\|f_{k}\right\|_{\alpha}\right)_{k \in \mathbb{N}}$ is uniformly bounded.

Note that

$$
f_{k}\left(\varphi\left(z_{k}\right)\right)=\frac{1-\left|\varphi\left(z_{k}\right)\right|^{2}}{\left(1-\varphi\left(z_{k}\right) \overline{\varphi\left(z_{k}\right)}\right)^{\alpha}}=\frac{1}{\left(1-\left|\varphi\left(z_{k}\right)\right|^{2}\right)^{\alpha-1}}
$$

Thus

$$
\begin{aligned}
\frac{\left|u\left(z_{k}\right)\right|\left(1-\left|z_{k}\right|^{2}\right)^{\beta}}{\left(1-\left|\varphi\left(z_{k}\right)\right|^{2}\right)^{\alpha-1}} & =\left(1-\left|z_{k}\right|^{2}\right)^{\beta}\left|u C_{\varphi} f_{k}\left(z_{k}\right)\right| \\
& \leq\left\|u C_{\varphi} f_{k}\right\|_{\beta} .
\end{aligned}
$$

Since $u C_{\varphi}: B^{\alpha} \rightarrow H_{\beta}^{\infty}$ is compact, it follow from the proof of the Weak Convergence Theorem in [9] that $\left\|u C_{\varphi} f_{k}\right\|_{\beta} \rightarrow 0$.Therefore

$$
\frac{\left|u\left(z_{k}\right)\right|\left(1-\left|z_{k}\right|^{2}\right)^{\beta}}{\left(1-\left|\varphi\left(z_{k}\right)\right|^{2}\right)^{\alpha-1}} \rightarrow 0
$$

as $k \rightarrow \infty$. So if $u C_{\varphi}$ is compact, then (2.2) holds.

\section{BOUNDEDNESS AND COMPACTNESS OF $u C_{\varphi}: B^{\alpha} \rightarrow H_{\beta}^{\infty}$ FOR $\alpha=1$}

In this section, we characterize the boundedness and compactness of $u C_{\varphi}: B^{\alpha} \rightarrow H_{\beta}^{\infty}$, when $\alpha=1$.

Theorem 3.3. Let $u$ be an analytic function on $\mathbb{D}, \varphi$ an analytic self-map of $\mathbb{D}, \alpha=1$ and $\beta$ a positive real number. Then $u C_{\varphi}: B^{\alpha} \rightarrow H_{\beta}^{\infty}$ is bounded if and only if

$$
\sup _{z \in \mathbb{D}}|u(z)|\left(1-|z|^{2}\right)^{\beta} \operatorname{Ln} \frac{e}{1-|\varphi(z)|^{2}}<\infty .
$$


Proof. First we obtain sufficiency. For a function $f \in B^{\alpha}$, we have

$$
\begin{aligned}
\sup _{z \in \mathbb{D}}\left(1-|z|^{2}\right)^{\beta}\left|u C_{\varphi} f(z)\right| & =\sup _{z \in \mathbb{D}}|u(z)|\left(1-|z|^{2}\right)^{\beta}|f(\varphi(z))| \\
& \leq \sup _{z \in \mathbb{D}}\left|u(z)\left(1-|z|^{2}\right)^{\beta} C\right| \mid f \|_{\alpha} \operatorname{Ln} \frac{e}{1-|\varphi(z)|^{2}} \\
& =C|| f \|_{\alpha} .
\end{aligned}
$$

In the above inequality we use the Lemma 1.1 for $\alpha=1$. Thus $u C_{\varphi}$ maps $B^{\alpha}$ boundedly into $H_{\beta}^{\infty}$.

Now, suppose that $u C_{\varphi}: B^{\alpha} \rightarrow H_{\beta}^{\infty}$ is bounded. For fixed $z_{0} \in \mathbb{D}$, consider the function $f_{0}$ defined by

$$
f_{0}(z)=\operatorname{Ln} \frac{e}{1-z \overline{\varphi\left(z_{0}\right)}}
$$

for $z \in \mathbb{D}$.

It is easy to check that $f_{0} \in B$, and then

$$
\begin{aligned}
|u(z)|\left(1-|z|^{2}\right)^{\beta} \mid \operatorname{Ln} \frac{e}{1-\varphi(z) \overline{\varphi\left(z_{0}\right)} \mid} & =|u(z)|\left(1-|z|^{2}\right)^{\beta}\left|f_{0}(\varphi(z))\right| \\
& =\left(1-|z|^{2}\right)^{\beta}\left|u C_{\varphi} f_{0}(z)\right| \\
& \leq\left\|u C_{\varphi} f_{0}\right\|_{\beta} \\
& \leq C\left\|f_{0}\right\|_{\alpha}<\infty .
\end{aligned}
$$

So for $z_{0} \in \mathbb{D}$, we have

$$
\left|u\left(z_{0}\right)\right|\left(1-\left|z_{0}\right|^{2}\right)^{\beta} \operatorname{Ln} \frac{e}{1-\left|\varphi\left(z_{0}\right)\right|^{2}}<\infty .
$$

Since $z_{0}$ is arbitrary, hence

$$
\sup _{z \in \mathbb{D}}|u(z)|\left(1-|z|^{2}\right)^{\beta} \operatorname{Ln} \frac{e}{1-|\varphi(z)|^{2}}<\infty .
$$

Theorem 3.4. Let $u$ be an analytic function on $\mathbb{D}, \varphi$ an analytic self-map of $\mathbb{D}, \alpha=1$ and $\beta$ a positive real number. Then $u C_{\varphi}: B^{\alpha} \rightarrow H_{\beta}^{\infty}$ is compact if and only if $u \in H_{\beta}^{\infty}$ and

$$
\lim _{|\varphi(z)| \rightarrow 1}|u(z)|\left(1-|z|^{2}\right)^{\beta} \operatorname{Ln} \frac{e}{1-|\varphi(z)|^{2}}=0 .
$$

Proof. Suppose that

$$
\lim _{|\varphi(z)| \rightarrow 1}|u(z)|\left(1-|z|^{2}\right)^{\beta} \operatorname{Ln} \frac{e}{1-|\varphi(z)|^{2}}=0 .
$$

By the assumption, for every $\varepsilon>0$, there is a $\delta \in(0,1)$ such that

$$
|u(z)|\left(1-|z|^{2}\right)^{\beta} \operatorname{Ln} \frac{e}{1-|\varphi(z)|^{2}}<\varepsilon
$$

whenever $\delta<|\varphi(z)|<1$. To prove the compactness of $u C_{\varphi}$, assume that $\left(f_{k}\right)_{k \in \mathbb{N}}$ is a bounded sequence in $B^{\alpha}$ such that $\left\|f_{k}\right\|_{\alpha} \leq 1$ and converges to zero uniformly on compact subsets of $\mathbb{D}$. We show that $\left\|u C_{\varphi} f_{k}\right\|_{\beta} \rightarrow 0$.

if $|\varphi(z)|>\delta$, then by (3.5),

$$
\begin{aligned}
\left\|u C_{\varphi} f_{k}\right\|_{\beta} & =\sup _{z \in \mathbb{D}}\left(1-|z|^{2}\right)^{\beta}\left|u C_{\varphi} f_{k}(z)\right| \\
& =\sup _{z \in \mathbb{D}}|u(z)|\left(1-|z|^{2}\right)^{\beta}|| f_{k} \|_{\alpha} L n \frac{e}{1-|\varphi(z)|^{2}} \\
& <\varepsilon\left\|f_{k}\right\|_{\alpha} \leq \varepsilon .
\end{aligned}
$$


Now consider $|\varphi(z)| \leq \delta$. In this case we have

$$
\left\|u C_{\varphi} f_{k}\right\|_{\beta}=\sup _{z \in \mathbb{D}}\left(1-|z|^{2}\right)^{\beta}\left|u C_{\varphi} f_{k}(z)\right| .
$$

Since $u \in H_{\beta}^{\infty}$, therefore $\left\|u C_{\varphi} f_{k}\right\|_{\beta} \rightarrow 0$.

We are going to prove that (3.4) is also necessary condition for compactness of $u C_{\varphi}$. Suppose that $\left(z_{k}\right)_{k \in \mathbb{N}}$ is a sequence in $\mathbb{D}$ such that $\left|\varphi\left(z_{k}\right)\right| \rightarrow 1$ as $k \rightarrow \infty$.

Consider the functions $f_{k}$ defined by

$$
f_{k}(z)=\frac{1}{\operatorname{Ln} \frac{e}{1-\left|\varphi\left(z_{k}\right)\right|^{2}}}\left(\operatorname{Ln} \frac{e}{1-z \overline{\varphi\left(z_{k}\right)}}\right)^{2} \quad \text { for } \quad z \in \mathbb{D} .
$$

Clearly $f_{k} \rightarrow 0$ uniformly on compact subset of $\mathbb{D}$, and

$$
\left|f_{k}^{\prime}(z)\right|=\frac{2}{\operatorname{Ln} \frac{e}{1-\left|\varphi\left(z_{n}\right)\right|^{2}}} \frac{\left|\overline{\varphi\left(z_{n}\right)}\right|}{\mid 1-z \overline{\varphi\left(z_{n}\right) \mid}} \cdot \operatorname{Ln} \frac{e}{\mid 1-z \overline{\varphi\left(z_{n}\right)}}<\infty .
$$

Therefore, $f_{k} \in B$ and $\left(\left\|f_{k}\right\|_{\alpha}\right)_{k \in \mathbb{N}}$ is uniformly bounded.

Not that

Thus

$$
f_{k}\left(\varphi\left(z_{n}\right)\right)=\operatorname{Ln} \frac{e}{1-\left|\varphi\left(z_{n}\right)\right|^{2}} .
$$

$$
\begin{aligned}
\left|u\left(z_{k}\right)\right|\left(1-\left|z_{k}\right|^{2}\right)^{\beta} \operatorname{Ln} \frac{e}{1-\left|\varphi\left(z_{n}\right)\right|^{2}} & =\left(1-\left|z_{k}\right|^{2}\right)^{\beta}\left|u C_{\varphi} f_{k}\left(z_{k}\right)\right| \\
& \leq\left\|u C_{\varphi} f_{k}\right\|_{\beta} .
\end{aligned}
$$

Since $u C_{\varphi}: B \rightarrow H_{\beta}^{\infty}$ is compact, it follows from the proof of the Weak Convergence Theorem in [9] that, $\left\|u C_{\varphi} f_{k}\right\|_{\beta} \rightarrow 0$. Therefore

$$
\left|u\left(z_{n}\right)\right|\left(1-\left|z_{k}\right|^{2}\right)^{\beta} \operatorname{Ln} \frac{e}{1-\left|\varphi\left(z_{n}\right)\right|^{2}} \rightarrow 0
$$

as $k \rightarrow \infty$. So, if $u C_{\varphi}$ is compact, then (3.4) holds.

4. BOUNDEDNESS AND COMPACTNESS OF $u C_{\varphi}: B^{\alpha} \rightarrow H_{\beta}^{\infty}$ FOR $0<\alpha<1$

In this section, we characterize the boundedness and compactness of $u C_{\varphi}: B^{\alpha} \rightarrow H_{\beta}^{\infty}$ when $0<\alpha<1$.

Theorem 4.5. Let $u$ be an analytic function on $\mathbb{D}, \varphi$ analytic self-map of $D, \beta$ a positive real number and $0<\alpha<1$. Then $u C_{\varphi}: B^{\alpha} \rightarrow H_{\beta}^{\infty}$ is bounded if and only if $u \in H_{\beta}^{\infty}$.

Proof. Suppose that $u \in H_{\beta}^{\infty}$. For a function $f \in B^{\alpha}$, we have

$$
\begin{aligned}
\sup _{z \in \mathbb{D}}\left(1-|z|^{2}\right)^{\beta}\left|u C_{\varphi} f(z)\right| & =\sup _{z \in \mathbb{D}}|u(z)|\left(1-|z|^{2}\right)^{\beta}|f(\varphi(z))| \\
& \leq \sup _{z \in \mathbb{D}}|u(z)|\left(1-|z|^{2}\right)^{\beta} C|| f \|_{\alpha} \\
& =C|| f \|_{\alpha} .
\end{aligned}
$$

In the above inequality we use the Lemma 1.1 for $\alpha \in(0,1)$. Thus $u C_{\varphi}$ maps $B^{\alpha}$ boundedly into $H_{\beta}^{\infty}$.

Now, suppose that $u C_{\varphi}$ maps $B^{\alpha}$ boundedly into $H_{\beta}^{\infty}$. Then $u=u C_{\varphi} 1 \in H_{\beta}^{\infty}$. This completes the proof of theorem.

Theorem 4.6. Let $u$ be an analytic function on $\mathbb{D}, \varphi$ analytic self-map of $\mathbb{D}, \beta$ a positive number and $0<\alpha<1$. Then $u C_{\varphi}: B^{\alpha} \rightarrow H_{\beta}^{\infty}$ is compact if and only if $u \in H_{\beta}^{\infty}$.

Before proving the above theorem, we need the following lemma, see [7]. 
Lemma 4.2. Let $0<\alpha<1$ and $T$ be a bounded linear operator from $B^{\alpha}$ into normed linear space $Y$. Then $T$ is compact if and only if $\left\|T f_{k}\right\|_{Y} \rightarrow 0$ whenever $\left(f_{k}\right)_{k \in \mathbb{N}}$ is a norm-bounded sequence in $B^{\alpha}$ that converges to 0 uniformly on $\overline{\mathbb{D}}$.

Proof of Theorem 4.6. We have already shown that $u \in H_{\beta}^{\infty}$ is necessary for the weighted composition operator $u C_{\varphi}: B^{\alpha} \rightarrow H_{\beta}^{\infty}$ to be bounded. Suppose that $f_{k} \in B^{\alpha}$ with $\left\|f_{k}\right\|_{\alpha} \leq 1$ for all $k=1,2, \ldots$ and $f_{k} \rightarrow 0$ uniformly on $\overline{\mathbb{D}}$.

Then

$$
\begin{aligned}
\sup _{z \in \mathbb{D}}\left(1-|z|^{2}\right)^{\beta}\left|u C_{\varphi} f_{k}(z)\right| & =\sup _{z \in \mathbb{D}}|u(z)|\left(1-|z|^{2}\right)^{\beta}\left|f_{k}(\varphi(z))\right| \\
& \leq\|u\|_{\beta} \sup _{|w| \leq 1}\left|f_{k}(w)\right| \rightarrow 0
\end{aligned}
$$

as $k \rightarrow \infty$. So, $\left\|u C_{\varphi} f_{k}\right\|_{\beta} \rightarrow 0$ as $k \rightarrow \infty$. It follows from lemma 4.2 that the operator $u C_{\varphi}$ maps $B^{\alpha}$ compactly into $H_{\beta}^{\infty}$.

Now, suppose that $u C_{\varphi}$ maps $B^{\alpha}$ compactly into $H_{\beta}^{\infty}$. Then $u=u C_{\varphi} 1 \in H_{\beta}^{\infty}$. This completes the proof of theorem (4.6).

\section{CONCLUSIONS}

Boundedness and compactness of (weighted) composition operators on different spaces of analytic functions are studied by many authors. This concepts for composition operators on Bers-type and between Bloch-type spaces were studied in [12] and [6]. We have studied the boundedness and compactness of weighted composition operators from Bloch-type to $n$th weighted-type spaces in [5]. Also, in [11], by using the hyperbolic analytic Besov-type classes, we investigate the boundedness and compactness of composition and weighted composition operators from Bloch-type to Besov-type spaces.

In this paper we give the necessary and sufficient conditions for boundedness and compactness of weighted composition operators from Bloch-type space $B^{\alpha}$ into Berse-type spaces in three cases, $\alpha>1, \alpha=1$ and $\alpha<1$. So, the methods and results of this paper are essentially different from [11].

\section{REFERENCES}

[1] Colonna, F. and Li, S., Weighted composition operator from the Besov spaces to the Bloch spaces, Bull. Malaysian Sci. Soc., 36 (2013), No. 4, 1027-1039

[2] Cowen, C. C. and Maccluer, B. D., Composition operators on spaces of analytic functions, Studies in Advanced Math., CRC Press, Boca Raton, 1995

[3] Hassanlou, M., Vaezi, H. and Wang, M., Weighted composition operators on weak vector-valued Bergman spaces and Hardy spaces, Banach J. Math. Anal., 9 (2015), No. 2, 35-43

[4] Kummar, S., Weighted composition operators between spaces of Dirichlet-Type, Rev. Math. Complut., 22 (2009), No. 2, 469-488

[5] Li, S., Abbasi, E. and Vaezi, H., Weighted composition operators from Bloch-type spaces to nth weighted-type spacese, Annales Polonici Mathematici, 124 (2020), No. 1, 93-107

[6] Lou, Z., Composition operators on Bloch-type spaces, Anal., 22 (2003), 81-95

[7] Ohno, S., Strorthoff, K. and Zhao, R., Weighted composition operators between Bloch-type spaces, Rocky mountain J. Math., 33 (2003), No. 1, 191-215

[8] Salaryan, A. and Vaezi, H., Adjoints of rationally induced weighted composition operators on the Hardy, Bergman and Dirichlet spaces, Period. Math. Hung., 72 (2016), 76-89

[9] Shapiro, J. H., Composition operators and classical function theory, Springer Verlag, New York, 1993

[10] Stevic, S., On an integral operator on the unit ball in $\mathbb{C}^{n}$, J. Inequal. Appl., 1 (2005), 81-88

[11] Vaezi, H. and Houdfar, S., Composition and weighted composition operators from Bloch-type to Besov-type spacese, Math. Reports, to appear.

[12] Weixian, H. and Lijian, J., Composition operator on Bers-type spaces, Acta. Math. Sci., 22B (2002), No. 3, 404412 
DEPARTMENT OF MATHEMATICS

FACULTY OF MATHEMATICAL SCIENCES

UNIVERSITY OF TABRIZ

TABRIZ, IRAN

Email address: hvaezi@tabriz.ac.ir

Email address: m.naghlisaretabrizu.ac.ir 